\title{
Numerical Modeling of a Turbulent Flow in a Flat Channel with a Fibrous Coating on the Walls
}

\author{
H. C. Sadouk ${ }^{1}$, S. Chikh ${ }^{2}$ and G. Lauriat ${ }^{3}$ \\ ${ }^{1}$ ESSA-Alger, Physics Faculty, Algiers, 16001, Algeria \\ ${ }^{2}$ USTHB, Faculty of Mechanical and Process Engineering, LTPMP, Algiers 16111, Algeria \\ ${ }^{3}$ Université Paris-Est, Laboratoire Modélisation et Simulation Multi Échelle, MSME FRE 3160 CNRS, Cité \\ Descartes, Champs-sur-M arne, 77454 Marne-la-Vallée Cedex 2, France
}

†Corresponding Author Email: hsadouk@hotmail.com

(Received April 12, 2018; accepted July 2, 2018)

\begin{abstract}
Turbulent forced convection heat transfer in a parallel plate channel partially or totally filled with a porous layer is studied numerically. The walls of the duct are non-permeable and heated uniformly at the same temperature. The present model adopts a single domain approach using continuity conditions at the interface. The governing equations (continuity, momentum, energy and $\kappa-\varepsilon$ turbulence model) are solved by applying the finite volume method. The Darcy-Brinkman-Forchheimer model is used in momentum equations, kinetic energy and turbulent dissipation rate using the $\kappa-\varepsilon$ turbulence model with adapted law of the wall. The obtained numerical results allow us to evaluate the effect of key parameters namely the Reynolds number, the thickness of the porous layer, its permeability, its porosity and the effective thermal conductivity on the flow and heat transfer. It is highlighted that the turbulent kinetic energy is strongly influenced by the permeability and the porous layer thickness. It is found that the presence of the porous medium substantially increases the heat transfer for an optimum thickness for very specific conditions.
\end{abstract}

Keywords: Heat transfer; $\kappa-\varepsilon$ model; Finite volume method; Parallel plates; Porous media.

\section{NOMENCLATURE}

$\begin{array}{ll}C_{F} & \text { forchheimer inertia coefficient } \\ C_{p} & \text { fluid specific heat } \\ D_{a} & \text { Darcy number } \\ D_{h} & \text { hydraulic diameter } \\ H & \text { channel height } \\ I & \text { turbulence intensity } \\ J & \text { viscosity ratio } \\ k & \text { thermal conductivity } \\ K & \text { permeability of the porous substrate } \\ L & \text { channel length } \\ N_{u} & \text { Nusselt number } \\ p & \text { pressure } \\ P_{r} & \text { Prandtl number } \\ R_{c} & \text { thermal conductivity ratio } \\ R_{e H} & \text { Reynolds number based on hydraulic } \\ & \text { diameter } \\ R_{e p} & \text { Reynolds number based on pore diameter } \\ T & \text { temperature } \\ U & \text { mean flow velocity } \\ U_{D} & \text { darcy velocity }\end{array}$

$\begin{array}{ll}\varepsilon & \text { dissipation rate of turbulent energy } \\ \kappa & \text { turbulent kinetic energy } \\ \rho & \text { density } \\ \lambda & \text { constant } \\ \phi & \text { porosité } \\ \sigma & \text { turbulent Prandtl number } \\ \mu & \text { dynamic Viscosity } \\ v & \text { kinematic viscosity } \\ \delta_{p} & \text { thickness layer } \\ \text { Subscripts } \\ \text { in } & \text { inlet of channel } \\ e f f & \text { effective } \\ a v & \text { average } \\ f & \text { fluid } \\ p & \text { porous medium } \\ w & \text { wall of channel } \\ s & \text { bulk (solid matrix) } \\ t & \text { turbulence } \\ C-f & \text { critical fluid region } \\ C-p & \text { critical fluid region }\end{array}$




\section{INTRODUCTION}

Heat transfer in porous media has many industrial and environmental applications, such as heat exchangers, chemical reactors or heat pipe technology. The insertion of a porous medium into a fluid channel efficiently improves the heat transfer performance because of the increase of the contact surface with the fluid. The porous medium alters the flow conditions yielding a thinner boundary layer and changes the heat conduction coefficient, which is generally higher than the fluid under investigation.

Two approaches have often been used to establish turbulence models in a porous medium. The first is based on the macroscopic Darcy-Forchheimer model. The second is based on averaged Reynolds equations. Both methods use the $\kappa-\varepsilon$ closure model. Silva and De Lemos (2003) used the second method and analyzed the influence of the interfacial condition between the fluid and the porous medium in a partially porous channel. Antohe and Lage (1997) concluded that the effect of the porous medium is to reduce the rate of turbulence, especially if the permeability is low. Kuznetsov et al. (2002) suggested that in most porous media, the turbulent flow in the fluid region is re-laminarized in the porous region. Getachew et al. (2000) developed a modified form of the $\kappa$ - $\varepsilon$ model for turbulence in porous media. Their approach is based on time averaging the Darcy Brinkman Forchheimer equation.

The presence of two regions of different natures in the same physical domain, a porous region through which flows a fluid and a clear fluid region. Both require the introduction of a fluid/porous interface to define the associated boundary conditions.

Silva and De Lemos (2003) presented a numerical study for a turbulent flow in a partially porous channel by introducing a new parameter, which represents the jump constraint. Note that the main consideration was to include the continuity of shear stress at the interface. Their assumption led to an intimate dependence between the characteristics of the porous medium and the jump constraint. Zhu and Kuznetsov (2005) assumed the absence of turbulence in the porous medium occurred when the fluid part has a high turbulence rate. Two models have been analyzed and compared (algebraic Cebeci-Smith method and $\kappa-\varepsilon$ ). The results showed that there is a significant difference between the values of the Nusselt number calculated by the two methods. De Lemos (2005) considered, in addition to the shear jump at the interface, the diffusional jump of $\kappa$. He proved that this assumption reflects more accurately the reality.

In the present study, the flow and heat transfer in a parallel plate channel partially or totally filled with a porous medium are analyzed. Governing equations are discretized and solved for both domains using a single numerical methodology. The $\kappa-\varepsilon$ turbulence model is used at the macroscopic scale. The effects of the porous matrix on the flow regime and heat transfer are analyzed and documented.

\section{MATHEMATICAL FORMULATION}

\subsection{Domain of Study}

The mathematical model is based on the transport equations for turbulent Newtonian fluid in a flat duct partially or totally filled by a porous medium. The walls are heated at a constant temperature $T_{w}=400 \mathrm{~K}$. The fluid (water) at the inlet of the channel has a constant velocity $U_{0}$ and is at a constant temperature $T_{0}=300 \mathrm{~K}$. The turbulence intensity at the inlet is set at $5 \%$. The length of the channel $L$ is chosen so that the flow is hydrodynamically and thermally fully developed at high Reynolds numbers. The thermodynamic properties of the fluid are taken at the average mixing temperature $T_{a v}=350 \mathrm{~K}$. The physical domain is schematically shown in Fig. 1.

It is assumed that the flow is in a steady state, incompressible and two-dimensional on average. The permeability and thermal conductivity of the porous medium are homogeneous and isotropic.

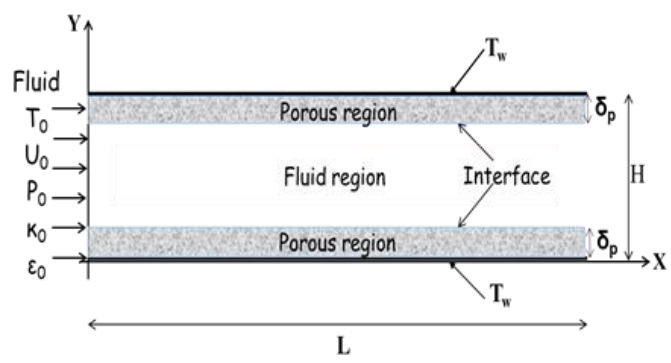

Fig. 1. Physical domain.

The conservation equations modeling the problem are described in Lage's work (1997). The closing coefficients used in the $\kappa-\varepsilon$ model are given by Hanjalic and Launder (1976) for clear medium $\left(\phi=1\right.$ and $\left.D_{a} \cdots \rightarrow \infty\right): C_{\mu}=0,09$, $C_{\epsilon 1}=1,44, C_{\epsilon 2}=1,92, \sigma_{k}=1,0, \sigma_{\varepsilon}=1,3$. The standard $\kappa-\varepsilon$ model is unsuitable for the region close to the wall and requires special treatment. The logarithmic law of the wall of Hanjalic and Launder (1976) is retained in this study.

As in the works of Allouache and Chikh (2008) and Chan et al. (2007), a single domain hybrid modeling is chosen. This approach allows avoiding the connection of conservation equations at the microscopic (fluid) and macroscopic (porous medium) scales at the fluid/porous interface. The writing of the connection conditions on either side of the interface remains indeed a subject of controversy (Chan et al., 2007).

\subsection{Governing Equations}

Given these assumptions, the transport equations inside a representative elementary volume $(R E V)$, written for convenience in tensor notation, as in Getachew et al. (2000) are cast in the following 
form:

\section{- Continuity Equation}

$\frac{\partial U_{i}}{\partial X_{i}}=0$

\section{- Momentum equations}

$$
\begin{gathered}
U_{j} \frac{\partial}{\partial X_{j}} U_{j}=-\frac{1}{\rho} \frac{\partial P}{\partial X_{i}}+\frac{\partial}{\partial X_{j}}\left[\left(v J+v_{t}\right) \frac{\partial U_{i}}{\partial X_{j}}\right] \\
+\frac{\partial}{\partial X_{j}} v_{t}\left[\frac{\partial U_{j}}{\partial X_{j}}\right]-\frac{2}{3} \frac{\partial \kappa}{\partial X_{j}}-\varnothing \frac{v}{\mathrm{~K}} U_{i} \\
-\phi^{2} \frac{C_{F}}{K^{1 / 2}}\left[-\frac{\left(U_{j} U_{j}\right)^{1 / 2} U_{i}}{\left(U_{j} U_{j}\right)^{1 / 2}}\left(v_{t}\left\{\frac{\partial U_{i}}{\partial X_{j}}+\frac{\partial U_{j}}{\partial X_{i}}\right\}-\frac{2}{3} \kappa\right)\right]
\end{gathered}
$$

\section{- Energy Equation}

$U_{j} \frac{\partial T}{\partial X_{j}}=\frac{\partial}{\partial X_{j}}\left[\left(\frac{1}{\phi} \frac{R_{c} \cdot v}{P_{r}}+\frac{v_{t}}{\sigma_{t}}\right) \frac{\partial T}{\partial X_{j}}\right]$

$v_{t}=C_{\mu} \kappa^{2} / \varepsilon$ is the turbulent viscosity.

$\sigma_{t}$ is the turbulent Prandtl number for temperature.

\section{- $\quad$ Equation of Turbulent Kinetic Energy}

$$
\begin{aligned}
& U_{k} \frac{\partial \kappa}{\partial X_{k}}=v_{t}\left[\frac{\partial U_{i}}{\partial X_{k}}+\frac{\partial U_{k}}{\partial X_{i}}\right] \frac{\partial U_{i}}{\partial X_{k}}+\frac{\partial}{\partial X_{k}}\left[v J+\frac{v_{t}}{\sigma_{k}}\right] \frac{\partial \kappa}{\partial X_{k}} \\
& -2 \phi \frac{v}{K} \kappa-J \varepsilon \\
& -\phi^{2} \frac{C_{F}}{\left(U_{k} U_{k}\right)^{1 / 2} K^{1 / 2}}\left[\begin{array}{c}
-v_{t}\left(\frac{\partial U_{i}}{\partial X_{k}}+\frac{\partial U_{k}}{\partial X_{i}}\right)\left(U_{k} U_{i}\right) \\
+\frac{8}{3} \kappa\left(U_{k} U_{k}\right) \\
+\frac{5}{3}\left(-\frac{4}{3} C_{s} U_{k} \frac{\kappa^{2}}{\varepsilon} \frac{\partial \kappa}{\partial X_{k}}\right) \\
+\frac{4}{3} C_{s} U_{k} \frac{\kappa^{2}}{\varepsilon} v_{t} \frac{\partial}{\partial X_{i}}\left(\frac{\partial U_{i}}{\partial X_{k}}+\frac{\partial U_{k}}{\partial X_{i}}\right)
\end{array}\right]
\end{aligned}
$$

$C_{s}$ is a constant of the diffusion model proposed by Mellor and Herriny (1973). $C_{s}=0.108$.

$\sigma_{\kappa}$ is the turbulent Prandtl number for kinetic energy.

\section{- Dissipation Rate Equation}

$$
\begin{aligned}
& U_{k} \frac{\partial \varepsilon}{\partial X_{k}}=\frac{\partial}{\partial X_{k}}\left[\left(v J+\frac{v_{t}}{\sigma_{\varepsilon}}\right) \frac{\partial \varepsilon}{\partial X_{k}}\right] \\
& +C_{\varepsilon 1} \frac{\varepsilon}{\kappa} v_{t}\left(\frac{\partial U_{i}}{\partial X_{j}}+\frac{\partial U_{j}}{\partial X_{i}}\right) \frac{\partial U_{i}}{\partial X_{j}}-J C_{\varepsilon 2} \frac{\varepsilon^{2}}{\kappa}-2 \phi \frac{v}{K} \epsilon
\end{aligned}
$$

$$
\left[\begin{array}{c}
\left(U_{k} U_{k}\right)^{1 / 2} \varepsilon+v\left(\frac{\partial}{\partial X_{j}}\left(U_{k} U_{k}\right)^{1 / 2}\right) \frac{\partial \kappa}{\partial X_{j}} \\
+\frac{v}{3} \frac{\partial}{\partial X_{j}} \frac{\left(U_{k} U_{i}\right)}{\left(U_{k} U_{k}\right)^{1 / 2}} \frac{\partial \kappa}{\partial X_{j}} \\
+\frac{U_{k}}{\left(U_{k} U_{k}\right)^{1 / 2}} C_{\varepsilon 3} v_{t} \frac{\kappa}{\varepsilon}\left(\frac{\partial U_{k}}{\partial X_{j}}+\frac{\partial U_{j}}{\partial X_{k}}\right) \frac{\partial \epsilon}{\partial X_{j}} \\
+\frac{1}{3} \frac{\left(U_{k} U_{i}\right)}{\left(U_{k} U_{k}\right)^{1 / 2} \varepsilon} \\
-\frac{v}{2} v_{t} \frac{\partial}{\partial X_{j}} \frac{\left(U_{k} U_{i}\right)}{\left(U_{k} U_{k}\right)^{1 / 2}}\left(\frac{\partial^{2} U_{i}}{\partial X_{j} \partial X_{k}}+\frac{\partial^{2} U_{k}}{\partial X_{j} \partial X_{i}}\right) \\
-\left(\frac{2}{3} \frac{U_{k}}{\left(U_{k} U_{k}\right)^{1 / 2}} C_{\varepsilon 3} \frac{\kappa}{\varepsilon} \kappa\right) \frac{\partial \epsilon}{\partial X_{j}}
\end{array}\right]
$$

$\sigma_{\varepsilon}$ is the turbulent Prandtl number for dissipation rate of turbulence kinetic energy.

\subsection{Boundary Conditions}

The imposed boundary conditions to complete the problem formulation are as follows:

- $\quad$ At the channel inlet $(\mathrm{x}=0)$ :

$$
\begin{aligned}
& U=U_{0}, V=0, T=T_{0} \\
& \kappa=\kappa_{0}=I U_{0}^{2}, \varepsilon=\varepsilon_{0}=\kappa_{0}^{3 / 2} / \lambda D_{h}, \lambda=0,33
\end{aligned}
$$

- $\quad$ At the exit of the channel $(x=L)$ : a fully developed flow and a negligible axial diffusion are imposed.

$\frac{\partial U}{\partial x}=0, \frac{\partial V}{\partial x}=0, \frac{\partial T}{\partial d x}=0, \frac{\partial \kappa}{\partial x}=0, \frac{\partial \varepsilon}{\partial x}=0$

- On solid walls:

$U=V=0, T=T_{w}, \kappa=0, \varepsilon=0$

The non-slip condition is applied to the impermeable wall.

- At the porous/fluid region $\left(x=\delta_{p}\right.$ and $x=$ $H-\delta_{p}$ ): For simplicity, the classical condition of continuity given by Allouache et al. (2008) is imposed on the fluid/porous interface on velocities, temperature, turbulent kinetic energy and the dissipation rate. The following interface conditions, therefore, need to be satisfied.

$U_{f}=U_{p}, \kappa_{f}=\kappa_{p}, \varepsilon_{f}=\varepsilon_{p}, T_{f}=T_{p}$

$\left.\left(\mu+\mu_{t}\right) \frac{\partial U}{\partial y}\right|_{f}=\left.\left(\mu_{e f f}+\mu_{t}\right) \frac{\partial U}{\partial y}\right|_{p}$

$\left.\left(\mu+\mu_{t}\right) \frac{\partial \kappa}{\partial y}\right|_{f}=\left.\left(\mu_{e f f}+\mu_{t}\right) \frac{\partial \kappa}{\partial y}\right|_{p}$

$\left.\left(\mu+\mu_{t}\right) \frac{\partial \varepsilon}{\partial y}\right|_{f}=\left.\left(\mu_{e f f}+\mu_{t}\right) \frac{\partial \epsilon}{\partial y}\right|_{p}$ 
Table 1 Comparison of the Nusselt number ( $\mathrm{Nu}$ ) for laminar flow

\begin{tabular}{|c|c|c|c|c|}
\hline & Meshing & Nusselt computed Present study & Nusselt Bejan, (1984) & Error (\%) \\
\hline Clear fluid channel & $50 \times 30$ & 7.54098 & 7.54 & 0.013 \\
\hline porous channel & $50 \times 30$ & 9.88207 & 9.87 & 0.122 \\
\hline
\end{tabular}

$$
\left.\frac{\partial T}{\partial y}\right|_{f}=\left.\left(\frac{R_{c} \mu}{\phi P_{r}}+\frac{\mu_{t}}{\sigma_{t}}\right) \frac{\partial T}{\partial y}\right|_{p}
$$

With the single domain approach, the following limit values of the parameters specific to the porous medium make it possible to define the type of the medium:

a) For a fluid medium:

$$
J=1, \rightarrow R_{c}=1, \phi=1 \text { and } K \rightarrow \infty
$$

b) For a porous medium:

$$
\begin{gathered}
J>1, \rightarrow R_{c} \neq 1, \phi<1 \text { and } \\
K \rightarrow \text { very low finitevalue }
\end{gathered}
$$

\section{NUMERICAL PROCEDURE}

The Navier-Stokes and Energy equations are solved on the orthogonal cartesian coordinate system using the finite volume method. The velocity components are stored on a staggered mesh. The convectiondiffusion terms are approximated using the QUICK (Quadratic Upwind Interpolation for Convective Kinematics) scheme. The SIMPLE iterative algorithm is used for the treatment of velocitypressure coupling and the line-by-line method is adopted in solving systems of algebraic equations.

Computations are carried out within half of the domain, assuming axial symmetry of the flow. After several trial computations to test the code sensitivity, a $100 \times 50$ mesh in $X$ and $Y$ coordinates respectively, allows satisfactory accuracy in hydrodynamically and thermally fully developed flow. The convergence criterion of the iterative process is given by the value of the residue, normalized by the flow rate of the variables at the inlet of the pipe, fixed at $10^{-4}$.

By applying the principle of mass flow conservation for a channel partially filled by a porous medium, the Reynolds number that defines the flow regime in the porous medium as a function of its permeability is written:

$$
R_{e_{i n}}=R_{e_{C-f}}+\frac{R_{e_{C-p}}}{\sqrt{D_{a}}} \frac{\mu_{e f f}}{\mu_{f}}
$$

Where $D_{a}$ is Darcy number based on the hydraulic diameter of the duct $\left(D_{a}=K / D_{h}{ }^{2}\right)$.

$\operatorname{Re}_{C-f} \approx 3000$ and $\operatorname{Re}_{C-p} \approx 300$ are the values of Reynolds number corresponding to the laminarturbulent transition in a clear fluid channel and in a duct filled with a porous medium respectively.

We consider the case of a constant viscosity $\left(\mu_{\text {eff }} / \mu_{f}=1\right)$, Eq. (17) allows us to plot the laminarturbulent transition curve in a porous medium for different values of its permeability. Two flow zones are distinguished from Fig. 2.

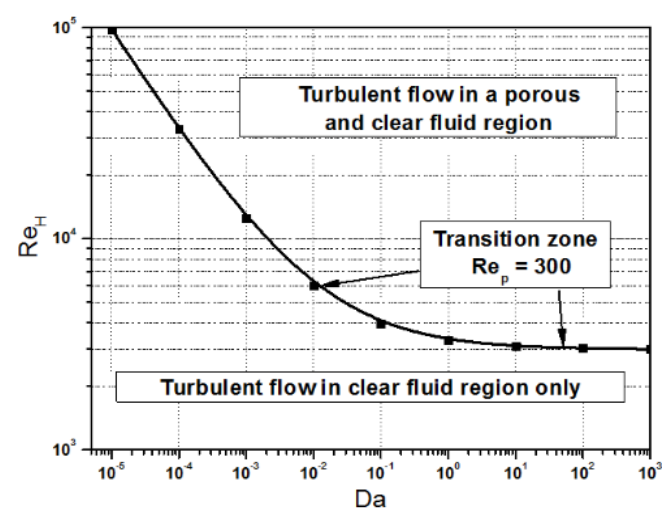

Fig. 2. Turbulent transition curve in the porous medium.

Above the curve, the flow is turbulent in both regions. Below, the flow is laminar in the porous region. The results of this plot allow choosing adequate conditions in order to achieve turbulence in the porous region.

\section{VALIDATION}

Comparisons are made in laminar and turbulent regimes for both cases of clear fluid channel and fully porous channel. A length $L=4 m$ and a height $H=0.02 \mathrm{~m}$ are considered for validation of the laminar flow case.

Table 1 gives a comparison of the computed values with the analytical solutions for fully developed laminar flow (Bejan, 1984). The relative deviation from the analytical solutions are found less than $0.2 \%$.

For turbulent flow conditions, the validation of the computational code is established for the porous limit case corresponding to clear fluid $(\phi=1$ and $\left.D_{a} \rightarrow \infty\right)$ The results are given in Table 2 .

The length of the channel is fixed to $L=6 \mathrm{~m}$ to ensure that the flow is dynamically and thermally established. The validation is done by comparison with the values of Nusselt number calculated from the correlations of Dittus-Boelter (1930) and Gnielinsky (1976). 
H. C. Sadouk et al. /JAFM, Vol. 12, Special Issue, pp. 1-8, 2019.

Table 2 Comparison of the Nusselt number $(\mathrm{Nu})$ for turbulent flow

\begin{tabular}{|c|c|c|}
\hline & Reference & ComputedNu \\
\hline Present study & & 195.7 \\
\hline \multirow{2}{*}{ Correlation } & Dittus - Boelter (1930) & 187.5 \\
\cline { 2 - 3 } & Gnielinski (1976) & 207.6 \\
\hline
\end{tabular}

The maximum relative differences vary between $2 \%$ and $6 \%$ for Reynolds numbers ranging from $10^{4}$ to $10^{5}$. A good agreement is observed for the both considered cases.

\section{RESULTS AND DISCUSSION}

The numerical study is carried out to investigate the forced convection heat transfer induced by a turbulent flow in a parallel plate channel partially or totally filled with a porous or fibrous material. The values used in the simulations are: $T_{0}=300 \mathrm{~K}$, $T_{w}=400 \mathrm{~K}, \quad k_{f}=0.665 \mathrm{~W} / \mathrm{m} / \mathrm{K}, \quad P_{r}=2.4 \quad$ (Water at $\left.T_{a v}=350 K\right), \quad k_{p} / k_{f}=100, \quad R_{c}=k_{\text {eff }} / k_{f}=10.9, \quad \mu_{\text {eff }} / \mu_{f}=1$, $C_{F}=0.1$.

To have fully developed conditions at the exit of the channel for different values of the Reynolds number, the values of the channel length and the distance between the plates are respectively taken equal to $10 \mathrm{~m}$ and $0.02 \mathrm{~m}$. The results are obtained at the exit of the channel. For the given axial symmetry, the spatial variations of the dynamic and thermal fields are represented on half of the height.

For a fully porous channel, the influence of permeability of the porous medium on the axial velocity profile is shown in Fig. 3. The results show that the velocity profile becomes more and more flattened for lower Darcy number. The velocity gradients increase near the walls because of the damping effect of the porous material and the shear stresses near the wall. We can notice that the axial velocity profile in the center part of the channel becomes relatively insensitive to the permeability when the Darcy number values exceed $10^{-3}$.

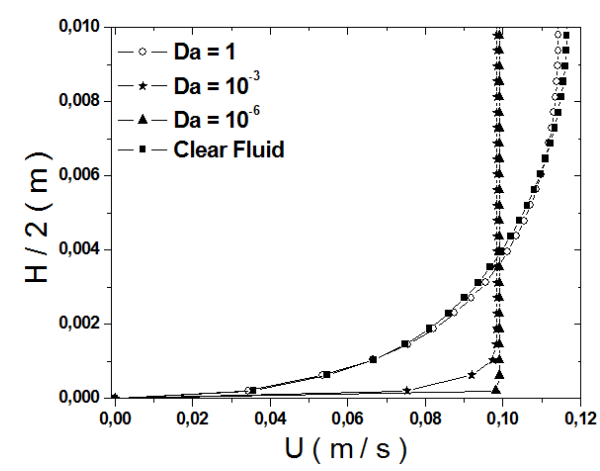

Fig. 3. Effect of Darcy number on the axial velocity profile at the exit of the fully porous channel (For $R_{e}=10^{4}, R_{c}=10.9$ ).

The effect of Reynolds number on the velocity profile at the exit section is shown in Fig. 4. Notice that for the same Darcy number, an increase in the Reynolds number at the inlet of the channel leads to an increase in the velocity magnitude. The velocity profile remains flat in the presence of the porous medium in the central part of the channel.

A peak of turbulent kinetic energy is observed in the near-wall region while the value of $\kappa$ is almost null in the central part of the channel as it is highlighted in Fig. 5. The porous medium increases the viscous and inertial forces and causes a greater friction compared to the clear fluid channel.

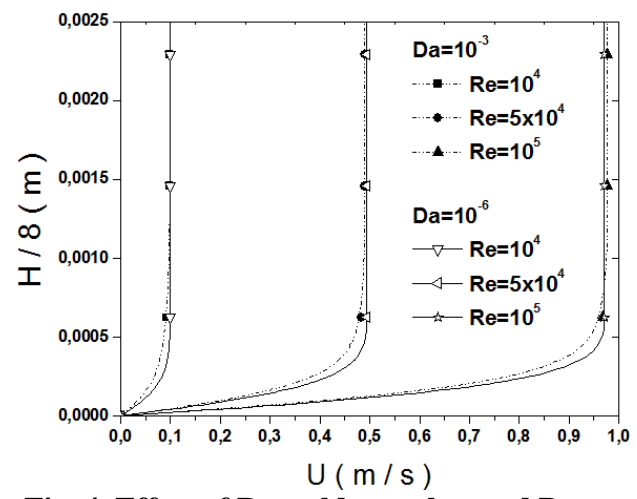

Fig. 4. Effect of Reynolds number and Darcy number on the velocity profile at the exit of the fully porous channel.

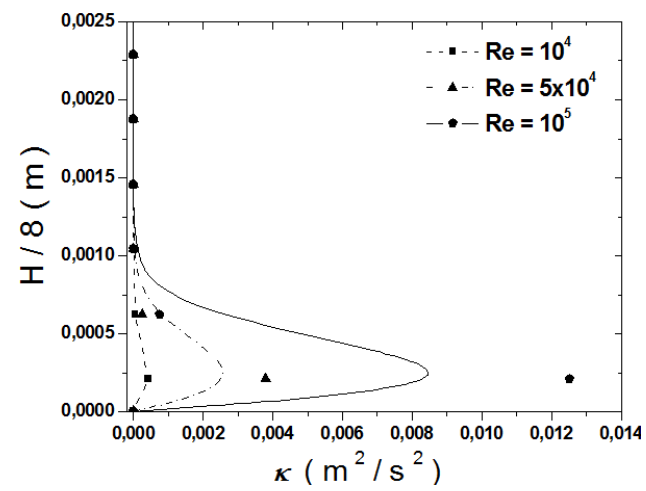

Fig. 5. Profile of turbulent kinetic energy at the exit of the fully porous channel (For $D_{a}=10^{-3}$, $R_{c}=10.9$ ).

The spatial evolution of the temperature shows that, for the same thermal conductivity ratio $\left(R_{c}=10.9\right)$, the temperature increases when the flow velocity in the channel is low. It is also noted that the temperature increases rapidly when the porous matrix conductivity is more important. These observations are shown in Fig. 6. 


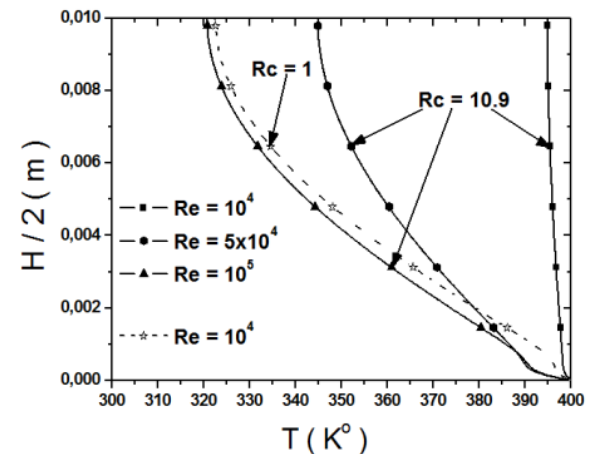

Fig. 6. Effect of Reynolds number and thermal conductivity ratio on the temperature profile at the exit of the fully porous channel (For $D_{a}=10^{-3}$ ).

For simulations related to channel partially filled by a porous medium, the evolution of the axial velocity and the turbulent kinetic energy for different thicknesses of the porous layer, occupying from $20 \%$ to $80 \%$ of the channel height, are studied.

Figure 7 shows the thickness effect of the porous layer on the velocity profile at the exit of the channel. For Reynolds number $\left(R_{e}=10^{4}\right)$ and Darcy number $\left(D_{a}=10^{-3}\right)$, we notice that the lower is the passage section of the clear fluid, the more important is the acceleration of the fluid in the central part of the channel. With the imposed flow rate $\left(R_{e}=10^{4}\right)$, the axial velocity increases up to $70 \%$ in the central part of the channel. The maximum velocity located in the central part (clear fluid region) increases proportionally with the thickness of porous medium. The fluid escapes from the porous matrix and moves towards the clear region in the center of the channel.

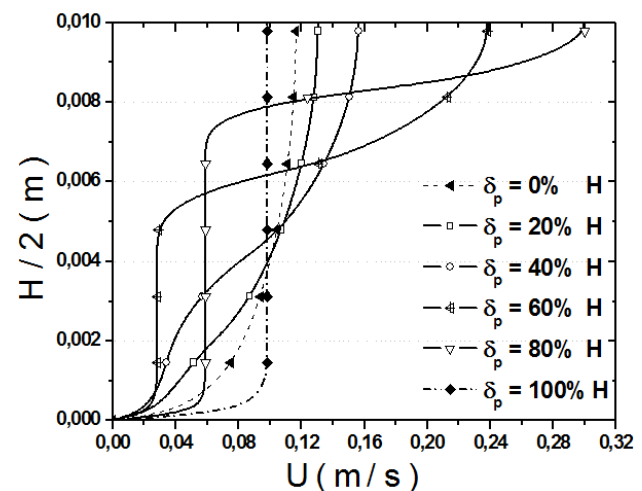

Fig. 7. Influence of the thickness of the porous layers on the axial velocity profile at the exit of the partly porous channel (For $\left.D_{a}=10^{-3}, R_{e}=10^{4}\right)$.

Figure 8 presents the evolution of turbulent kinetic energy for different thicknesses of the porous layer, occupying from $20 \%$ to $80 \%$ of the channel height. When the thickness of the porous medium increases, the turbulent kinetic energy increases in the fluid zone and reaches its maximum at the zone near the fluid/porous interface. Although the section of the fluid region decreases, the fluid flow increases which causes an increase in the coefficient of friction in the fluid/porous interface which becomes important. The inertial effects and the strength of the porous medium cause a strong kinetic energy gradient.

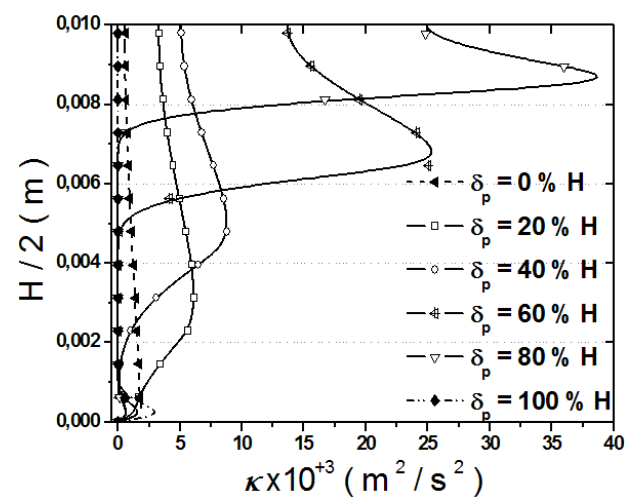

Fig. 8. Influence of the thickness of the porous layers on the turbulent kinetic energy profile at the exit of the partly porous channel (For $D_{a}=10^{-}$ $\left.{ }^{3}, R_{e}=10^{4}\right)$.

According to the work of Boubendir and Chikh (2009), the maximum production of turbulent kinetic energy has been obtained for small thicknesses. The analysis of the effect of the thickness of the porous substrate has shown the existence of optimal and critical thicknesses (thickness close to $20 \%$ of the channel height) for which we have a maximum heat transfer.

Therefore, in the following results, we present the case where the porous layer occupies $20 \%$ of the channel height.

The simulation results in Fig. 9 show that the permeability and porosity of the porous medium have a significant influence on the velocity profile. The decrease of the permeability leads to a greater flow rate flowing in the clear fluid region and consequently the maximum value of velocity is reached in the center of channel. Moreover, a reduction of the porosity yields a lower volume fraction within the porous layer and causes a decrease in velocity.

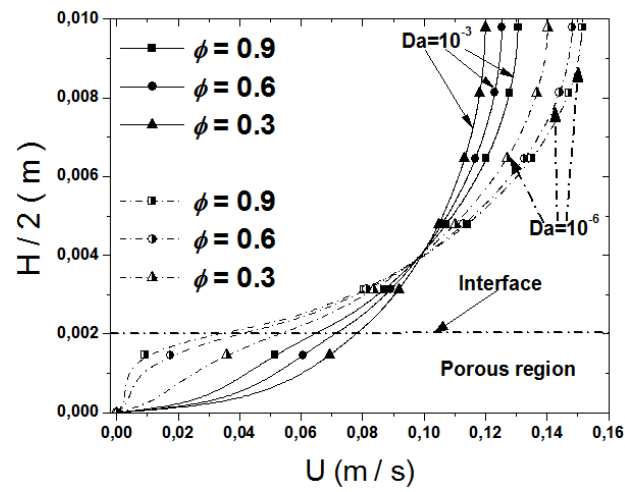

Fig. 9. Effect of Darcy number and porosity on axial velocity at the exit of the partly porous channel (For $R_{e}=10^{4}, \delta_{p}=20 \% \mathrm{H}$ ).

Figure 10 shows the effect of the Darcy number and porosity on the temperature profile. Decreasing the Darcy number decreases the pore- 
scale fluid velocity, resulting in a significant decrease in the temperature. We notice a clear decrease in temperature when the porosity decreases. For constant permeability the effect of porosity greatly affects the temperature of the fluid. The lower the porosity; the higher the thermal conductivity ratio is, which causes an increase in the temperatures.

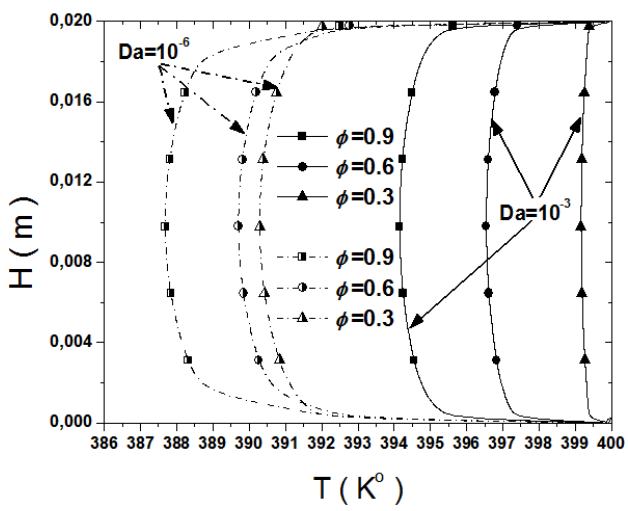

Fig. 10. Effect of Darcy number and porosity on the temperature profile at the exit of the partly porous channel (For $\left.R_{e}=10^{4}, \delta_{p}=20 \% \mathrm{H}\right)$.

To analyze the effect of the thermal conductivity ratio on heat transfer, a parametric study was carried out in order to follow the evolution of the average temperature and the Nusselt number established according to different conductivity ratios for different permeability values of the porous layer. The simulation results are illustrated in Fig. 11 and Fig. 12 respectively.

Notice that in Fig. 11, a considerable increase in the average temperature of the fluid at the outlet section of the channel. This increase is controlled by the increase in the thermal conductivity ratio. For a value of $R_{c}=10.9$, the fluid temperature at the exit of the channel reaches a maximum increase of $10 \%$ compared to the case of the ratio $R_{c}=1$. The temperature continues to increase to reach an asymptotic value. The influence of the $R_{c}$ shows the existence of an optimal ratio of thermal conductivity for which the temperature reaches its maximum.

Figure 12 shows the variation of the Nusselt number established for different ratios of thermal conductivity for different permeability values of the porous medium. The results show that increasing the thermal conductivity of the porous substrate makes it possible to increase the Nusselt number which follows a quasi-exponential law before stabilizing towards an asymptotic value. The increase in the Nusselt number corresponds to a significant improvement in heat transfer to reach an optimum value beyond which the heat transfer reaches its maximum. A clear improvement is noted compared to the fully fluid case for conductivity ratio values beyond $R_{c}=10$. Heat transfer is significantly improved with lower permeability. When the porous medium is more permeable the heat exchange becomes higher.

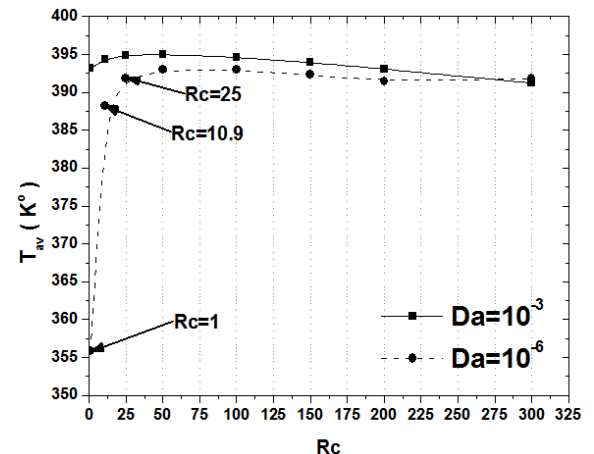

Fig. 11. Influence of the effective thermal conductivity on the average temperature at the exit of the partly porous channel (For $\left.R_{e}=10^{4}\right)$.

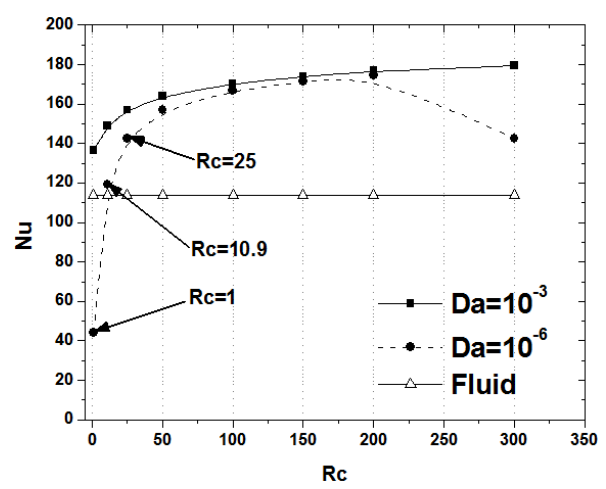

Fig. 12. Influence of the effective thermal conductivity on the local Nusselt number at the exit of the partly porous channel (For $\left.R_{e}=10^{4}\right)$.

\section{CONCLUSION}

The present work is a numerical study of heat transfer for a turbulent flow in a channel partially or filled by a porous substrate. The single domain approach is applied in the numerical implementation and the continuous conditions at the fluid/porous interface are applied. To improve the heat transfer with the presence of a porous medium, the choice of the Reynolds number, the thickness of the porous layer, its permeability, porosity, effective thermal conductivity will play an important role on the flow and on the heat exchange in the channel. The maximum of turbulent kinetic energy is observed in the region near the fluid/porous interface and is strongly influenced by inertial and shear effects. This highly sensitive area requires a refined treatment with adaptive laws.

The increase in the ratio of thermal conductivities improves considerably the heat transfer and allows a maximum of heat exchange. In addition to the optimum value of the thermal conductivity ratio, the improvement of the heat exchange depends on permeability of the porous layer. A maximum production of turbulent kinetic energy has been obtained for small thicknesses of the porous layer.

The results show that the mathematical model developed in this study makes it possible to predict the thermal and dynamic characteristics of a turbulent flow in a channel partially or filled with a porous medium. 
H. C. Sadouk et al. /JAFM, Vol. 12, Special Issue, pp. 1-8, 2019.

\section{ACKNOWLEDGEMENTS}

The first author thanks the Laboratory of Polyphasic Transport and Porous Media (LTPMP, USTHB) and the Lab. Modélisation et Simulation Multi Echelle (MSME, MLV) for supporting this research and all researchers of these laboratories for their helpful comments.

\section{REFERENCES}

Allouache, N. and S. Chikh (2008). Numerical modeling of turbulent flow in an annular heat exchanger partly filled with a porous substrate. Journal of Porous Media 7, 617-632.

Antohe, B. V. and J. L. Lage (1997). International journal of Heat and Mass Transfer 40, 30133024.

Bejan, A. (1984). Convection Heat Transfer, Wiley, New York.

Boubendir, L. and S. Chikh (2009). Analyse de l'effet de la matrice poreuse sur le transfert thermique en régime turbulent dans un échangeur à plaques, Revue des Énergies Renouvelables 12(1), 45-53.

Chan, H. C., W. C. Huang, J. M. Leu and C. J. Lai (2007). Macroscopic modeling of turbulent flow over a porous medium. International Journal of Heat and Fluid Flow 28, 11571166.

De Lemos, M. J. S. (2005). Turbulent kinetic energy distribution across the interface between a porous medium and a clear region. International Communications in Heat and Mass Transfer 32, 107-115.
Dittus, F. W. and L. M. K. Boelter (1930). Heat transfer in automobile radiators of the tubular type, Univ. Calif. Pub. Eng., 13, 443-461.

Getachew, D., W. J. Minkowycz and J. L. Lage (2000). A Modified of the $\kappa-\varepsilon$ model for turbulent flows of an incompressible fluid in porous media. International journal of Heat and Mass transfer 43, 2909-2915.

Gnielinsky, V. (1976). New equations for heat mass transfer in turbulent pipe channel flow. International Chemistry Engineering 16(2), 359-368.

G. L. Mellor and H. J. Herring (1973). A survey of mean turbulent field closure models. AIAA J, $11,590-599$.

Hanjalic K., Launder B.E (1976), Contribution towards a Reynolds-stress closure for lowReynolds-number turbulence, J. Fluid Mech. 74(4), 593-610.

Kuznetsov, A.V., L. Cheng and M. Xiong (2002). Numerical Heat transfer Part A: Applications 42, 365-383.

Silva, R.A. and M. J. S. De Lemos (2003). Turbulent flow in a channel occupied by a porous layer considering the stress jump at the interface. International journal of Heat and Mass transfer 46, 5113-5121.

Zhu, J. and A.V. Kuznetsov (2005). Forced convection in a composite parallel plate channel: modeling the effect of interface roughness and turbulence utilizing a $k-\varepsilon$ model. International communications in heat and mass transfer 32(1-2), 10-18. 